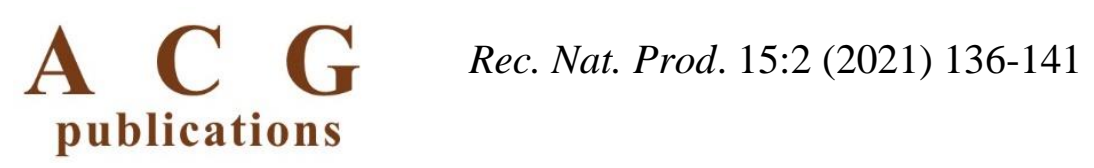

records of natural products

\title{
Novel Pharmacological Effects of Syringin on Anxiety Behavior and Autonomic Nervous System Activity
}

\section{Shouhei Miyazaki $\oplus^{1}$, Hirotaka Oikawa $\oplus^{2}$, Hideo Takekoshi $\oplus^{3}$, Masako Hoshizaki ${ }^{3}$, Masato Ogata ${ }^{4}$ and Takahiko Fujikawa ${ }^{1,2,4 *}$}

\author{
${ }^{1}$ Laboratory of Molecular Prophylaxis and Pharmacology, Graduate School of Pharmaceutical \\ Sciences, Suzuka University of Medical Science, 3500-3 Minamitamagaki-cho, Mie 513- \\ 8670, Japan \\ ${ }^{2}$ Faculty of Pharmaceutical Sciences, Suzuka University of Medical Science, 3500-3 \\ Minamitamagaki-cho, Mie 513-8670, Japan \\ ${ }^{3}$ Sun Chlorella Corp., Production \& Development Department, 369 Osaka-cho, Karasuma-dori Gojo- \\ sagaru, Shimogyo-ku, Kyoto 600-8177, Japan \\ ${ }^{4}$ Department of Biochemistry and Proteomics, Mie University Graduate School of Medicine, 2-174 \\ Edobashi, Tsu, Mie 514-8507, Japan
}

(Received August 22, 2020; Revised September 14, 2020; Accepted September 16, 2020)

\begin{abstract}
Acanthopanax senticosus HARMS (ASH), a traditional Chinese herbal medicine, has been used to treat arthralgi, muscle weakness of the lower body, hypertension, and impotence. ASH was also reported to increase resistance to stress and fatigue. Recently, the efficacy of ASH against depression and stress-induced gastric ulcer was demonstrated in a rodent model. Previously, we also reported that ASH exerted anxiolytic effects and modulated autonomic nervous system activity under stress. However, no research has examined the anxiolytic effects of isofraxidin and syringin, two constituents of ASH. Thus, we investigated the anxiolytic constituents of ASH. The rats were administered distilled water, isofraxidin or syringin for 1 week. Autonomic nervous system activity was estimated using heart rate variability and anxiety behavior in the improved elevated beam walking (IEBW) test. The syringin increased the time spent in the open arm in the IEBW test. The syringin blocked the significant decrease in normalized high-frequency power, indicative of parasympathetic nervous system activity, in the IEBW test. The isofraxidin exerted no significant effects on behavior and autonomic nervous system activity. These results indicated that syringin partially contributes to the anxiolytic and autonomic nervous systemmodulating effects of ASH.
\end{abstract}

Keywords: Acanthopanax senticosus; HARMS; isofraxidin; syringin; anxiolytics; autonomic nervous. () 2020 ACG Publications. All rights reserved.

* Corresponding author: E-Mail: fujikawa@suzuka-u.ac.jp; Phone: +81-59-340-0595 


\section{Plant Source}

A dried 2-5 cm root tip of Acanthopanax senticosus HARMS (ASH) from Heilongiiang, China, was cut and extracted with hot water. The extract was concentrated under reduced pressure and dried using a spray dryer. The herb specimen was authenticated by Professor Sansei Nishibe of the Laboratory of Pharmacognosy, Department of Pharmaceutical Sciences, Health Science University of Hokkaido. The extract powder was provided by Sun Chlorella Co., Ltd. (Kyoto, Japan) and a voucher specimen is deposited in Sun Chlorella Corp as LN 8142. Isofraxidin ( $\geq 99.0 \%, 099-03651$ FUJIFILM Wako Pure Chemical Corporation, Osaka, Japan) and syringin $(\geq 97 \%$, S920050, Toronto Research Chemicals, Toronto, Canada) were used.

\section{Previous studies}

Acanthopanax senticosus HARMS (ASH) is a deciduous shrub of the ragweed family that grows abundantly in various regions of Russia, China, Korea, and Northern Japan. ASH is described in classic herbalism literature such as Shennong Ben Cao Jing and Compendium of Materia Medica. ASH has been used in China for more than 2000 years to treat conditions such as rheumatic arthritis and neuralgia [1]. Recently, ASH was identified as an adaptogen [2]. Furthermore, ASH is also reported to increase resistance to stress and fatigue [3,4]. ASH is widely used as an alternative or complimentary medicine in Western countries based on its various pharmacological benefits and limited side effects [5]. ASH has a protective effect against gastric ulcers [3] and prevents depressive symptoms induced by tail suspension and the forced swimming test [4,6]. ASH was also reported to affect neurons [7-9], including protective effects against neurodegenerative disorders (such as Parkinson's disease) in rodents [10,11]. We previously reported that ASH attenuates the activation of sympathetic nervous system activity and the suppression of parasympathetic nervous system activity in stressful environments [12]. Recent studies identified bioactive components in ASH extract, including chlorogenic acid, eleutheroside E, isofraxidin (1), and, syringin (2) described their pharmacological effects $[1,13,14]$ (the structure in Figure 1). Various pharmacological effects of isofraxidin (1) were summarized previously [15]. In particular, anti-inflammatory and anti-oxidant effects were investigated in isofraxidin (1). Syringin (2) (also termed eleutheroside B) is considered to have immunomodulatory effect [16] and improve insulin resistance via acetylcholine release [17].<smiles>COc1cc2ccc(=O)oc2c(OC)c1O</smiles>

1<smiles>COc1cc(/C=C/CO)cc(OC)c1O[C@@H]1O[C@H](CO)[C@@H](O)[C@H](O)[C@H]1O</smiles>

2

Figure 1. The structure of isofraxidin (1) and syringin (2)

\section{Present Study}

The components in ASH responsible for its anxiolytic effects have not been identified. The major components of the ASH extract were measured by HPLC in our previous study [12]. In that study 
we have determined the main compounds of the hot water extract of the species as isofraxidin (1), eleutheroside B (2, syringin), B1 and E [12]. However, no research in behavioral pharmacology has examined the anxiolytic effects of compounds $\mathbf{1}$ and $\mathbf{2}$. In this study, we investigated the effect of compounds $\mathbf{1}$ and $\mathbf{2}$ on anxiety behavior and autonomic nervous system (ANS) activity in the improved elevated beam walking (IEBW) test which is the improved version of the elevated plus maze (EPM) in the point of the reproducibility and the variability in the ANS activity and the behavior.

We used the IEBW apparatus by installing a $2 \times 4$ timber $(180 \mathrm{~cm} \times 8.9 \mathrm{~cm}) 190 \mathrm{~cm}$ above the floor level, and the apparatus included open $(140 \mathrm{~cm} \times 8.9 \mathrm{~cm})$ and closed $(40 \mathrm{~cm} \times 8.9 \mathrm{~cm} \times 28.5 \mathrm{~cm})$ arms (see reference [12] and supporting information of article for details). Rats were placed on the tip of the open arm, then allowed to explore the area freely for $3 \mathrm{~min}$. We analyzed the time spent on the open-arm and cardiac ANS activity by HRV analysis. Each doses were decided by the consumption of the $5 \%(\mathrm{w} / \mathrm{w})$ ASH extract contained foods in the previous study [14]. The animal study was permitted by the Ethics Committee on Animal Use of the Suzuka University of Medical Science and conducted according to the "Guide for the Care and Use of Laboratory Animals (NIH Publication)" (see supporting information).

A

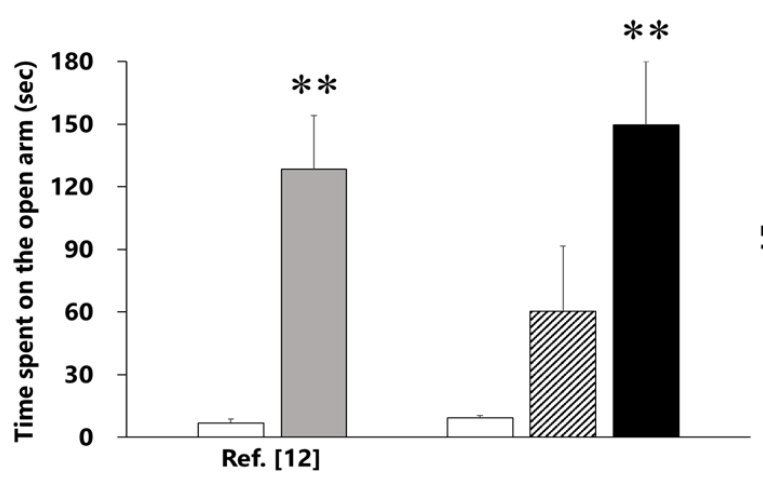

C

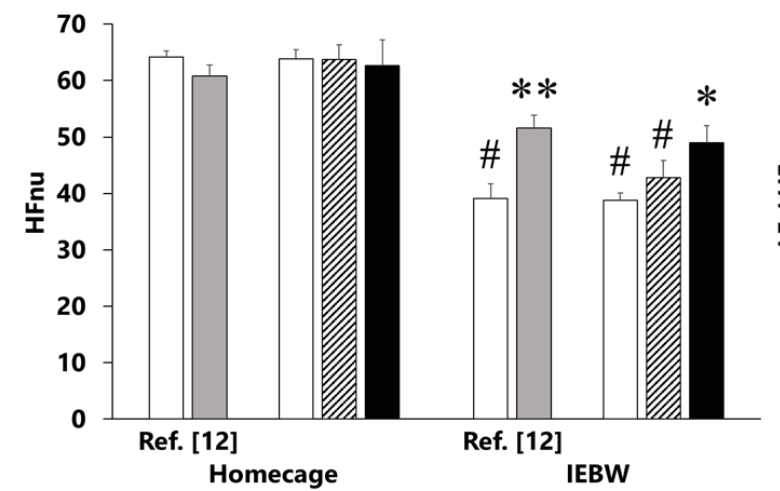

B

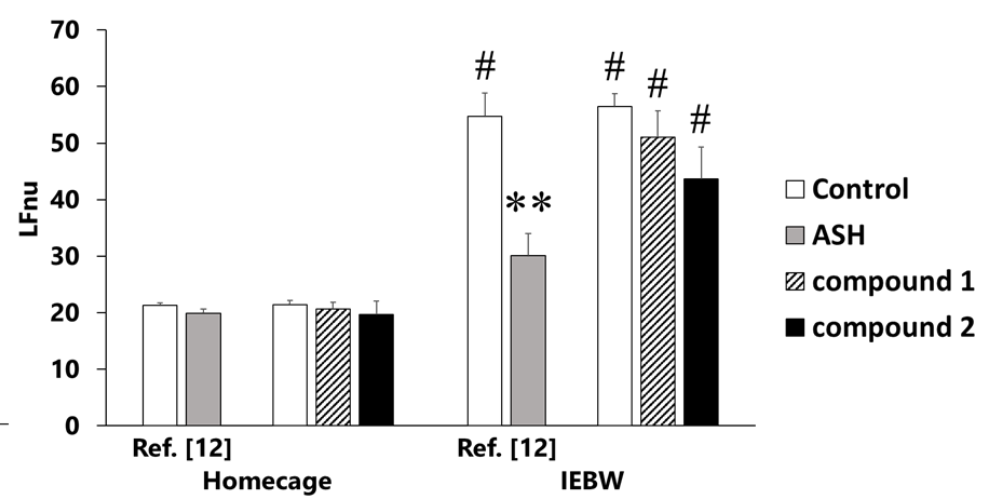

D

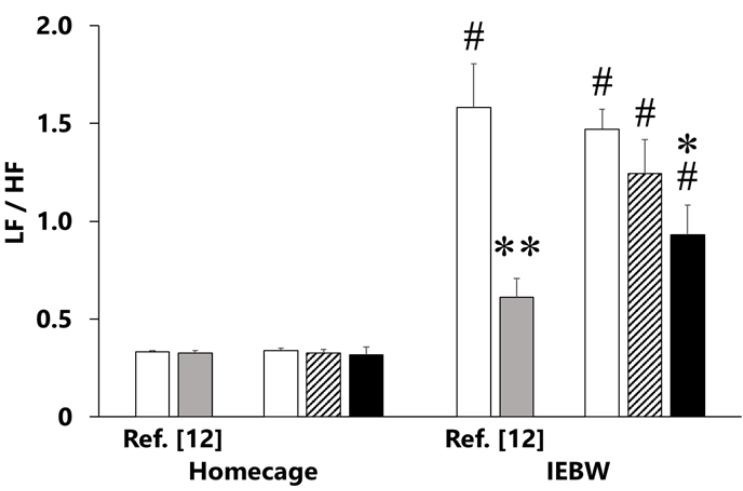

Figure 2. Effects of isofraxidin (1) and syringin (2) on the anxious behaviors and the cardiac autonomic nervous activity in the improved elevated beam walking test. (A) The time spent on the open arm. (B) Normalized unit of low-frequency (LF) power (LFnu) reflects sympathetic nervous system activity. (C) Normalized unit of high-frequency (HF) power (HFnu) reflects parasympathetic nervous system activity. (D) LF/HF indicates the balance between sympathetic and parasympathetic nervous system activities. Cont (left) and ASH5\% data is cited from our previous study [12]. Data are presented as the mean \pm standard error; $\mathrm{n}=5 ; * \mathrm{P}$ $<0.05, * * \mathrm{P}<0.01 \mathrm{vs}$. the Cont (left or right) group (Dunnett's or unpaired t-test), ${ }^{*} \mathrm{P}<0.05$ vs. each home cage (paired t-test). 
The time spent on the open arm was significantly increased in the compound 2 group compared with that in the Control group (Figure 2A). Compound 1-administered rats also exhibited a longer time spent on the open arm, but the difference was not significant. We placed rats implanted with a telemeter under home cage housing conditions and measured ANS activity at rest. No differences in ANS activity and the ratio of low-frequency power to high-frequency power (LF/HF) under home cage housing conditions were observed within any group (Figure 2B-D). Subsequently, we measured ANS activity under the IEBW conditions. The IEBW conditions significantly increased normalized unit of lowfrequency (LFnu) power and decreased normalized unit of high-frequency (HFnu) power in the Control and compound 1 groups compared with the findings under home cage conditions (Figure 2B and C). Additionally, we also observed significantly increased LF/HF in the Control and compound 1 groups under the IEBW conditions (Figure 2C). Under the IEBW conditions, the compound 2 exhibited significantly higher LFnu and LF/HF than those under home cage housing conditions. The administration of compound 2 under IEBW conditions suppressed the changes of HFnu and LF/HF observed in the Control group (Figure 2C and D).

Previous research revealed that the administration of ASH extract prolonged the time spent in the open arm of our previously developed IEBW apparatus (Figure 2A). ASH extract also attenuated the activation of the sympathetic nervous system and suppression of the parasympathetic nervous system. (Figure 2B-D) [12]. Prior reports illustrated that isofraxidin (1) exerted neuroprotective effects against amyloid beta-induced toxicity and inhibited monoamine oxidase B (MAOB) activity [15], suggesting benefits against anxiety behavior. Contrary to expectations, compound $\mathbf{1}$ did not significantly alter anxiety behavior and ANS activity in this study. The MAOB-inhibitory effect of isofraxidin (1) identified by Yan et al. using affinity ultrafiltration mass spectrometry was not evaluated in vivo [15]. The dose of compound $\mathbf{1}$ was determined based on the amount contained the ASH extract which showed anxiolytic effect in our previous study [12], but may not be appropriate for inhibiting MAOB activity. It was also reported that the concentration of isofraxidin (1) were different between the pure isofraxidin (1) and ASH extract treatment [18]. As the reason for that, the conversion from Eleutheroside B1 which is the precursor of isofraxidin (1) was considered by the authors. Additionally, the pharmacokinetic parameters of isofraxidin (1) are also different between the pure isofraxidin (1) and the ASH extract administration. For these reasons, further behavioral researches are needed to evaluate the anxiolytic effects of compound 1. Conversely, compound $\mathbf{2}$ administration attenuated the suppression of parasympathetic nervous system activity and prolonged the time spent in the open arm of the IEBW apparatus. Syringin (2) was reported to enhance the release of acetylcholine from nerve terminals. It is possible that the acetylcholine release-promoting activity of syringin (2) is related to increased parasympathetic nervous system activity in the IEBW test. Furthermore, oral syringin (2) administration also reduced nitric oxide (NO) concentrations and nitric oxide synthase (NOS) activity in the brains of mice [19], whereas research using RAW 264.7 mouse macrophages revealed no significant changes in NO production following treatment of syringin (2) [16]. Additionally, muscarinic receptor activation is known to induce NO production [20]. NO plays an important role in stress responses, and it is considered the therapeutic target [21]. Low doses of NOS inhibitors such as N $\omega$-nitro-L-arginine methyl ester (LNAME) and 7-nitroindazole exerted anxiolytic effects in the EPM test [22]. L-NAME also increased cardiac parasympathetic tone without affecting cardiac sympathetic tone [23]. However, NO itself is known to suppress sympathetic outflow and facilitate parasympathetic outflow [24]. Because the effects of NOS inhibitors and NO on ANS activity differ among studies because of the methodological conditions, $\mathrm{NO}$ was indicated to affect ANS activity by interacting with other factors. Thus, the effects of syringin (2) on NOS/NO may involve its promotion of acetylcholine release. The anxiolytic and ANSmodulatory effects of it uncovered in this study may be involved both the NOS/NO pathway and the promotion of acetylcholine release. Further research is needed to clarify the pharmacological mechanism.

Additionally, it is reported that the pharmacokinetic parameters of each pure ASH component are different from that of ASH extract administration [25]. Thus, the further researches regarding the pharmacological effect of the combination of each ASH component are needed. 
As a conclusion, taken together, it is considered that syringin (2) partially contributed to the anxiolytic effect of ASH, whereas the contribution of isofraxidin (1) might be in minimal level. The effects of syringin (2) may be related to its effects on the NOS/NO pathway and acetylcholine release.

\section{Acknowledgments}

This study was performed with support from Sun Chlorella Corp. (Kyoto, Japan). ASH extract was provided by Sun Chlorella Corp. The funders had no role in the design of the study; in the collection, analyses, or interpretation of data; in the writing of the manuscript, or in the decision to publish the results. The authors are grateful to M. Nakagawa for technical assistance and S. Nakao for animal care. The authors would like to thank Enago (www.enago.jp) for the English language review.

\section{Supporting Information}

Supporting Information accompanies this paper on http://www.acgpubs.org/journal/recordsof-natural-products

\section{ORCID}

Shouhei Miyazaki: 0000-0001-8810-8144

Hirotaka Oikawa: 0000-0002-2050-4957

Hideo Takekoshi: 0000-0003-1995-283X

Masako Hoshizaki: 0000-0003-1336-9977

Masato Ogata: 0000-0003-3516-653X

Takahiko Fujikawa: 0000-0003-3787-3422

\section{References}

[1] L. Z. Huang, H. F. Zhao, B. K. Huang, C. J. Zheng, W. Peng and L. P. Qin (2011). Acanthopanax senticosus: Review of botany, chemistry and pharmacology, Pharmazie 66, 83-97.

[2] I. I. Brekhman and I. V. Dardymov (1969). New substances of plant origin which increase nonspecific resistance, Annu. Rev. Pharmacol. 9, 419-430.

[3] T. Fujikawa, A. Yamaguchi, I. Morita, H. Takeda and S. Nishibe (1996). Protective effects of Acanthopanax senticosus Harms from Hokkaido and its components on gastric ulcer in restrained cold water stressed rats, Biol. Pharm. Bull. 19, 1227-1230.

[4] S. Nishibe, H. Kinoshita, H. Takeda and G. Okano (1990). Phenolic compounds from stem bark of Acanthopanax senticosus and their pharmacological effect in chronic swimming stressed rats, Chem. Pharm. Bull. (Tokyo). 38, 1763-1765.

[5] WHO (2004). WHO Monographs on Selected Medicinal Plants.

[6] B. Gaire and D. Lim (2014). Antidepressant effects of Radix et Caulis Acanthopanacis Santicosi extracts on rat models with depression in terms of immobile behavior, J. Tradit. Chinese Med (Chung $i$ tsa chih ying wen pan) 34, 317-323.

[7] S. Zhang, X. Li, Y. Wang, N. Zhang, Z. Yang, S. Liu and F. Lu (2014). Neuroprotection or neurotoxicity? New insights into the effects of Acanthopanax senticosus harms on nervous system through cerebral metabolomics analysis, J. Ethnopharmacol. 156, 290-300.

[8] F. Wu, H. Li, L. Zhao, X. Li, J. You, Q. Jiang, S. Li, L. Jin and Y. Xu (2013). Protective effects of aqueous extract from Acanthopanax senticosus against corticosterone-induced neurotoxicity in PC12 cells, $J$. Ethnopharmacol. 148, 861-868.

[9] T. Fujikawa, H. Soya, H. Hibasami, H. Kawashima, H. Takeda, S. Nishibe and K. Nakashima (2002). Effect of Acanthopanax senticosus Harms on biogenic monoamine levels in the rat brain, Phytother. Res. 16, 474-478.

[10] T. Fujikawa, S. Miguchi, N. Kanada, N. Nakai, M. Ogata, I. Suzuki and K. Nakashima (2005). Acanthopanax senticosus Harms as a prophylactic for MPTP-induced Parkinson's disease in rats, $J$. Ethnopharmacol. 97, 375-381.

[11] T. Fujikawa, N. Kanada, A. Shimada, M. Ogata, I. Suzuki, I. Hayashi and K. Nakashima (2005). Effect of sesamin in Acanthopanax senticosus HARMS on behavioral dysfunction in rotenone-induced 
[12] S. Miyazaki, H. Oikawa, H. Takekoshi, M. Hoshizaki, M. Ogata and T. Fujikawa, (2018). Anxiolytic effects of Acanthopanax senticosus HARMS occur via regulation of autonomic function and activate hippocampal BDNF-TrkB signaling, Molecules 24 (15 pages).

[13] T. Li, K. Ferns, Z. Q.Yan, S. Y. Yin, J. J. Kou, D. Li, Z. Zeng, L. Yin, X. Wang, H. X. Bao, Y. J. Zhou, Q. H. Li, Z. Y. Zhao, H. Liu and S. L. Liu (2016). Acanthopanax senticosus, photochemistry and anticancer potential, Am. J. Chin. Med. 44, 1543-1558.

[14] T. Deyama, S. Nishibe and Y. Nakazawa (2001). Constituents and pharmacological effects of Eucommia and Siberian ginseng, Acta Pharmacol. Sin. 22, 1057-1070.

[15] M.B. Majnooni, S. Fakhri, Y. Shokoohinia, M. Mojarrab, S. Kazemi-Afrakoti and M.H. Farzaei (2020). Isofraxidin: synthesis, biosynthesis, isolation, pharmacokinetic and pharmacological properties, Molecules 25 (23 pages).

[16] H. Sun, H. Lv, Y. Zhang, X. Wang, K. Bi and H. Cao (2007). Pharmacokinetics of isofraxidin in rat plasma after oral administration of the extract of Acanthopanax senticosus using HPLC with solid phase extraction method, Chem. Pharm. Bull. (Tokyo). 55(9):1291-1295.

[17] J.Y. Cho, K.H. Nam, A.R. Kim, J. Park, E.S. Yoo, K.U. Baik, Y.H. Yu and M.H. Park (2001). In-vitro and in-vivo immunomodulatory effects of syringin, J. Pharm. Pharmacol. 53, 1287-1294.

[18] K.Y. Liu, Y.C. Wu, I.M. Liu, W.C. Yu and J.T. Cheng (2008). Release of acetylcholine by syringin, an active principle of Eleutherococcus senticosus, to raise insulin secretion in Wistar rats, Neurosci. Lett. 434, 195-199.

[19] Y. Cui, Y. Zhang and G. Liu (2015). Syringin may exert sleep-potentiating effects through the NOS/NO pathway, Fundam. Clin. Pharmacol. 29, 178-184.

[20] H.C. Saternos, D.A. Almarghalani, H.M. Gibson, M.A. Meqdad, R.B. Antypas, A. Lingireddy and W.A. AbouAlaiwi (2018). Distribution and function of the muscarinic receptor subtypes in the cardiovascular system, Physiol. Genom. 50, 1-9.

[21] A. Kumar and P. Chanana (2017). Role of Nitric Oxide in Stress-Induced Anxiety. In Vitamins and Hormones, Academic Press Inc.Vol. 103, pp. 147-167.

[22] A. Spiaccijr, F. Kanamaru, F. Guimaraes and R. Oliveira (2008). Nitric oxide-mediated anxiolytic-like and antidepressant-like effects in animal models of anxiety and depression, Pharmacol. Biochem. Behav. 88, 247-255.

[23] K.E. Scrogin, D.C. Hatton, Y. Chi and F.C. Luft (1998). Chronic nitric oxide inhibition with 1 -NAME: effects on autonomic control of the cardiovascular system, Am. J. Physiol. Integr. Comp. Physiol. 274, R367-R374.

[24] H. D. Schultz (2009). Nitric oxide regulation of autonomic function in heart failure, Curr. Heart Fail. Rep. 6, 71-80.

[25] H. X. Fan, Z. P. Deng, H. Zhong, X. T. Xu and Q. Q. Yao (2014). Comparative pharmacokinetics of syringin, eleutheroside $\mathrm{E}$ and isofraxidin in rat plasma after intravenous administration of each monomer and Ciwujia injection (in Chinese), Zhongguo Zhongyao Zazhi (China J. Chin. Materia Med.), 39(10), 1921-1927.

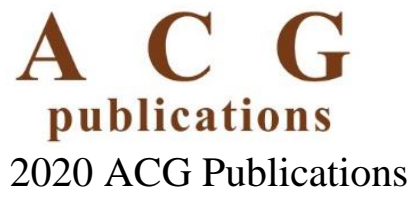

